\title{
Blessures chez les enfants : le Canada obtient une note médiocre
}

Publié sur www.jamc.ca le 2I septembre 2006.

$\mathrm{D}$ ans ce numéro, Birken et ses coauteurs documentent une baisse importante, de I97I à I998, du taux de blessures accidentelles chez les enfants du Canada vivant en milieu urbain (voir page 867$)^{1}$. Ils déterminent aussi que, dans le cas de ces blessures, l'écart des taux chez les enfants riches et les enfants pauvres ne s'est heureusement pas creusé. Néanmoins, les enfants pauvres demeurent deux fois plus susceptibles que ceux des familles nanties de mourir d'une blessure accidentelle. Il faut au moins reconnaître le pourcentage de cette strate qui comprend des groupes vulnérables (p. ex., Premières nations et enfants de réfugiés) et admettre que la question mérite d'être approfondie davantage qu'il est possible de le faire dans cet éditorial.

L'écart entre les taux de mortalité attribuables à des blessures chez les enfants riches et les enfant pauvres peut provoquer l'indignation morale, mais il faut garder une partie de cette indignation pour le rendement général du Canada au niveau du contrôle des blessures chez les enfants. Dans le deuxième Rapport Innocenti de l'UNICEF, qui porte avant tout sur la mortalité infantile selon le type de blessure, le Canada vient au $18^{\mathrm{e}}$ rang sur 26 pays de l'OCDE pour les décès causés par des blessures intentionnelles et accidentelles chez les enfants de I à I4 ans au cours de la période de I99I à I995². Le Canada s'est classé bien en-dessous des chefs de file mondiaux que sont les pays de la Scandinavie, ce qui n'est pas étonnant, mais il a aussi obtenu des résultats inférieurs à ceux de l'Espagne, de la Grèce et de l'Australie. Si le Canada avait obtenu les mêmes résultats que la Suède, qui se classe au premier rang, 2665 enfants de plus seraient vivants aujourd'hui.

Birken et ses coauteurs ${ }^{1}$ suggèrent des interventions ciblées pour refermer l'écart des taux de mortalité attribuables aux blessures entre les enfants riches et les enfants pauvres. Dans un commentaire connexe, Howard ${ }^{3}$ préconise de promouvoir davantage la prévention des blessures (voir page 899). La Société canadienne de pédiatrie a adopté cette dernière stratégie de promotion de la politique publique avec un succès particulier lorsqu'elle a publié son premier rapport sur la politique publique du Canada et la santé des enfants et des adolescents ${ }^{4}$. Dans ce rapport, la société met en évidence les politiques publiques autant bonnes que douteuses sur les blessures chez les enfants. Elle insiste sur les problèmes reliés à la circulation comme les casques de cycliste, les sièges d'appoint, les véhicules tout-terrain et les permis progressifs. Le rapport établit des paramètres permettant à chacun d'évaluer la situation dans sa propre région et, avec les éditions à venir, de suivre les progrès. Le rapport met en évidence les anomalies et les incohérences au niveau des politiques publiques sur la santé, ainsi que les possibilités et les réussites. Par exemple, une province a un excellent programme de permis progressifs, mais comme il n'y a pas de règlement, des enfants de deux ans peuvent conduire légalement des véhicules hors-route. Une autre province obtient de bons résultats face à ces mesures énumérées de contrôle des blessures, mais un résultat médiocre en matière de législation sur le casque de cycliste. L'adoption générale de la meilleure législation serait bénéfique pour tous les enfants du Canada. Une telle approche est axée sur le changement environnemental de façon à réduire le nombre des blessures au lieu de compter uniquement sur des programmes qui visent le comportement de la personne ou de la famille. Dans le contexte de cette approche environnementale générale, je suggère non seulement que nous envisagions des interventions qui visent les en- fants pauvres, mais que nous examinions aussi l'élément du contexte social qui est à l'origine de la disparité des taux de mortalité attribuables aux blessures - la pauvreté chez les enfants. En décembre I989, dans le dernier discours qu'il a prononcé au Parlement comme chef du Nouveau parti démocratique, Ed Broadbent a proposé une motion qui engageait le gouvernement fédéral à mettre fin à la pauvreté chez les enfants au Canada avant $2000^{5}$. La motion a été adoptée à l'unanimité, y compris par le premier ministre de l'époque, Brian Mulroney. Même après un changement du parti au pouvoir, Lloyd Axworthy du gouvernement Chrétien a réaffirmé l'engagement de son administration ${ }^{6}$. Dans un rapport sur la pauvreté chez les enfants ${ }^{7}$, le Centre de recherche Innocenti de l'UNICEF a toutefois classé le Canada au $\mathrm{I}^{\mathrm{e}}$ rang sur les 26 pays de l'OCDE et a constaté qu'on y avait réalisé peu ou pas du tout de progrès en I5 ans sur le plan de la réduction du taux de pauvreté chez les enfants.

Si les classements mondiaux du Canada quant au taux de blessures et de pauvreté chez les enfants se ressemblent tellement, c'est peut-être une coïncidence. Si le Canada déployait des efforts concertés et finalement fructueux pour réduire la pauvreté chez les enfants, notre place parmi les pays de l'OCDE devrait toutefois s'améliorer dans les futurs bulletins de l'UNICEF. Il faut néanmoins intervenir spécifiquement pour lutter sur divers fronts contre les blessures chez les enfants, depuis la création d'une stratégie fédérale sur la prévention des blessures ${ }^{1}$ jusqu'à l'adoption de politiques publiques saines aux échelons local, provincial et fédéral ${ }^{3}$. Globalement, le Canada ne devrait viser rien de moins que l'excellence pour tous les aspects de la santé des enfants.

\section{Richard Stanwick \\ Administration de la santé de l'île de Vancouver \\ Victoria (C.B.)}

Cet article a fait l'objet d'un examen par les pairs.

\section{RÉFÉRENCES}

I. Birken CS, Parkin PC, To T, et al. Trends in rates of death from unintentional injury among Canadian children in urban areas: influence of socioeconomic status. JAMC 2006;175(8):867-8

2. A league table of child deaths by injury in rich nations [Rapport Innocenti $\mathrm{n}^{\circ} 2$ ]. Florence : Centre de recherche Innocenti de l'UNICEF; 200I. Disponible à : www.uniceficdc.org/publications/pdf/repcard2e.pdf\#search=\%22A\%2oLeague\%2oTable\%2oof \%20Child\%20Deaths\%2oby\%2oInjury\%20in\%20Rich\%20Nations\%22 (consulté le I4 septembre 2006).

3. Howard AW. Injury in childhood: a vexingly simple problem [éditorial]. JAMC 2006; I75(8):899-900.

4. Société canadienne de pédiatrie. En faisons-nous assez? Un rapport de la situation des politiques publiques canadiennes de la santé des enfants et des adolescents. Ottawa : Société canadienne de pédiatrie; 2005. Disponible à : www.cps.ca/francais /proadv/DefenseDInterets/Rapport_Situation.pdf (consulté le I3 septembre 2006).

5. Hansard. Débats de la Chambre des communes. Disponible à : http://parlı .parl.gc.ca/36/I/parlbus/chambus/house/debates/I79_I999-02-II/hanI79_I6Io-f.htm (consulté le I3 septembre 2006).

6. Hansard. Débats de la Chambre des communes. Disponible à : www2.parl.gc.ca / HousePublications $/$ Publication.aspx?pub=hansard\&mee $=99 \&$ parl $=35 \&$ ses $=$ I \& Language $=\mathrm{F}$ (consulté le $\mathrm{I} 3$ septembre 2006).

7. La pauvreté des enfants dans les pays riches, 2005 [Rapport Innocenti no 6]. Florence : Centre de recherché Innocenti de l'UNICEF; 2005. Disponible à : www .unicef.org/brazil/repcard6f.pdf\#search=\%22Child\%2oPoverty $\% 20$ in $\% 20$ Rich\%20 Countries\%202005.\%22 (consulté le I3 septembre 2006). 